\title{
15
}

\section{FLASH: removing chemical barriers for volume immunofluorescence of whole intact organs}

Lorge Almagro ${ }^{1}$, Hendrik Messal $^{2}$, Axel Behrens $^{1}$ ${ }^{1}$ The Francis Crick Institute, United Kingdom. ${ }^{2}$ Netherlands Cancer Institute, Netherlands

\section{Abstract Text}

The appreciation of whole organs and organisms through optical clearing and fluorescence volume imaging allows the observation of tissues and cells in their native 3D context. This new dimension in imaging of biological samples has advanced our understanding of tissue morphogenesis and architecture in different fields of biomedical sciences. Most tissue clearing techniques were developed originally by neuroscience labs, and optimised for imaging the brain. However, the brain is a soft low-density organ, very different to other organs in its physical characteristics and chemical composition. Different procedures are required for achieving transparency and imaging other organs.

FLASH (Fast Light-microscopic analysis of Antibody-Stained wHole organs) is a novel technique developed in our lab for permeabilisation, clearing and immunofluorescence of most mammalian organs. FLASH consists of non-degradative epitope recovery and membrane solubilisation steps, with adaptations for different tissue characteristics (pigmentation, density, size...), and can be used to detect a multitude of antigens in disparate tissues. Retrieval and immunolabelling of epithelial markers, an obstacle for previous clearing techniques, can be achieved with FLASH. A single sample cleared by FLASH can be imaged in the light-sheet fluorescent microscope, obtaining a panoramic view of the entire organ, as well as in the confocal, discerning cellular and subcellular structures across the depth of the tissue. Moreover, FLASH preserves the integrity and architecture of the tissues, fully integrating the procedure with down-stream conventional histopathology when 
necessary.

Hence, FLASH is a versatile and accessible technique, and has been applied by us and other labs to answer a broad range of questions. FLASH has allowed us to visualise epithelial organs, investigate the mechanisms of tumour initiation, study the biophysics of mechanical tension in cancer morphogenesis and characterise neurodevelopmental defects in whole mouse embryos. We are working to expand the reach of FLASH, answering questions in a broader range of biomedical fields such as organoid imaging, neuroscience, immunology and clinical research. 